\title{
Directly Reprogrammed Neurons Express MAPT and APP Splice Variants Pertinent to Ageing and Neurodegeneration
}

\author{
Mette Habekost ${ }^{1,2}$ (D) Per Qvist ${ }^{1,3,4} \cdot$ Mark Denham $^{1,2} \cdot$ Ida E. Holm $^{5,6} \cdot$ Arne Lund Jørgensen ${ }^{1}$
}

Received: 29 July 2020 / Accepted: 9 December 2020 / Published online: 7 January 2021

(C) The Author(s) 2021

\begin{abstract}
Neurons produced by reprogramming of other cell types are used to study cellular mechanisms of age-related neurodegenerative diseases. To model Alzheimer's disease and other tauopathies, it is essential that alternative splicing of the MAPT transcript in these neurons produces the relevant tau isoforms. Human neurons derived from induced pluripotent stem cells, however, express tau isoform compositions characteristic of foetal neurons rather than of adult neurons unless cultured in vitro for extended time periods. In this study, we characterised the dynamics of the MAPT and APP alternative splicing during a developmental timecourse of porcine and murine cerebral cortices. We found age-dependent and species-specific isoform composition of MAPT, including $3 \mathrm{R}$ and $4 \mathrm{R}$ isoforms in the porcine adult brain similar to that of the adult human brain. We converted adult and embryonic fibroblasts directly into induced neurons and found similar developmental patterns of isoform composition, notably, the $3 \mathrm{R}$ and $4 \mathrm{R}$ isoforms relevant to the pathogenesis of Alzheimer's disease. Also, we observed cell-type-specific isoform expression of $A P P$ transcripts during the conversion. The approach was further used to generate induced neurons from transgenic pigs carrying Alzheimer's disease-causing mutations. We show that such neurons authentically model the first crucial steps in AD pathogenesis.
\end{abstract}

Keywords Induced neurons $\cdot$ Splicing $\cdot$ Tau $\cdot$ APP $\cdot$ Alzheimer's disease $\cdot$ Porcine models

\section{Introduction}

Neurodegenerative diseases classified as tauopathies have in common intraneuronal accumulation of different isoforms of

Mette Habekost

mhab@dandrite.au.dk

$\triangle$ Arne Lund Jørgensen

alj@biomed.au.dk

1 Department of Biomedicine, Aarhus University, 8000C Aarhus, Denmark

2 Danish Research Institute of Translational Neuroscience, Nordic EMBL Partnership for Molecular Medicine, Aarhus University, 8000C Aarhus, Denmark

3 iPSYCH, Lundbeck Foundation Initiative for Integrative Psychiatric Research, 8000C Aarhus, Denmark

4 Center for Genomics and Personalized Medicine, 8000C Aarhus, Denmark

5 Department of Pathology, Randers Hospital, 8930 Randers, Denmark

6 Department of Clinical Medicine, Aarhus University, 8000C Aarhus, Denmark the microtubules associated protein tau (MAPT) characteristic of each disease. Alzheimer's disease (AD) is a tauopathy where age-related neurodegeneration results in progressive dementia. The neuropathological findings include characteristic extraneuronal deposits in neuritic plaques of the amyloidogenic $A \beta$ fragment, development of intracellular neurofibrillary tangles (NFTs) of, in particular, tau isoforms with three and four microtubules-binding repeats $(3 R, 4 R)$, and neuronal death [1-3]. The pathogenic sequence of events is still a matter of dispute, but a long-held hypothesis points to increased production or decreased clearance of the $A \beta$ fragment from the brain as a prime driver of $A \beta$ deposition and subsequent NFT pathology $[4,5]$.

The vast majority of $\mathrm{AD}$ cases have multifactorial aetiology, and more than 50 susceptibility loci with genomewide significance are associated with the disease [6, 7]. In few cases, the disease behaves in an autosomal dominant fashion and is associated with mutations in the amyloid precursor protein $(A P P)$ or the presenilin (PSEN1 or PSEN2) genes [8-10].

APP is an integral membrane protein expressed in many cell types. Three main isoforms exist due to alternative splicing and encode proteins of 695, 751 and 770 amino acids 
(APP695, APP751 and APP770) with APP695 being the typical isoform in neurons $[11,12]$. Aberrant processing of APP by the proteolytic enzymes, $\beta$ - and $\gamma$-secretases generates $A \beta$, and mutations in $A P P$ and in $P S E N 1 / 2$ increase the production of $A \beta$ or create more of the hydrophobic 42 amino acids long $A \beta$ peptide $(A \beta 42)$ relative to the shorter $A \beta 40[13,14]$.

Tau belongs to the family of microtubule-associated proteins that also includes MAP2. Six isoforms of tau are produced by alternative splicing of MAPT. These isoforms differ by presence or absence of protein domains encoded by exon 2 and exon 3 in the $\mathrm{N}$-terminal part $(0 \mathrm{~N}, 1 \mathrm{~N}, 2 \mathrm{~N})$, and inclusion $(4 \mathrm{R})$ or exclusion $(3 \mathrm{R})$ of a microtubule-binding repeat encoded by exon 10 in the C-terminal part of tau [15]. Alternative splicing of MAPT is developmentally regulated, and not conserved between species. During human development, only $0 \mathrm{~N} 3 \mathrm{R}$ tau is expressed, whereas $3 R$ and $4 R$ tau isoforms are expressed at equal levels in the adult brain $[16$, 17]. Among mammals, only $4 \mathrm{R}$ tau is present in the adult rodent brain while both $3 R$ and $4 R$ tau isoforms appear to be expressed in the adult porcine brain $[18,19]$.

Typically, animal models of $\mathrm{AD}$ are generated in mice that have been genetically modified by introducing disease-causing mutations in APP or PSEN1/2. Such animals are expected to model both the autosomal dominant form and the multifactorial form of $\mathrm{AD}$ since the two forms of $\mathrm{AD}$ are indistinguishable with respect to clinical and neuropathological phenotypes. However, a serious drawback of these models has been their resistance to develop tau pathology in the form of NFT in the brain. This lack of a key neuronal lesion may be explained by the absence of $3 \mathrm{R}$ tau in mouse neurons since the tau isoform composition appears to be critical for filament formation $[3,20]$.

Human-induced pluripotent stem cells (iPSCs) serve as an attractive cellular alternative as this technology allows patientspecific neurons to be derived from fibroblasts. Indeed, neurons derived from iPSCs that originate from the fibroblasts of patients with $\mathrm{AD}$-causing mutations exhibit aberrant $\mathrm{A} \beta$ production [21]. However, it is a challenge to recapitulate tau isoform composition and hence pathology in such cells in vitro. Indeed, several studies have demonstrated the presence of foetal-only (0N3R) tau in wild-type iPSC-derived neurons during in vitro differentiation [22-24], although neurons with frontotemporal dementia (FTDP-17T)-causing $M A P T$ mutations mature and induce $4 \mathrm{R}$ tau expression faster than control neurons [25]. Accelerated maturation and adult tau splicing have also been achieved in wild-type neurons by enhancing the microenvironment using 3D culturing or in vivo transplantation $[26,27]$. In this study, we characterise the dynamics of $M A P T$ and $A P P$ alternative splicing during porcine and murine embryonic brain development and demonstrate that directly reprogrammed porcine neurons model agedependent and cell-type-specific patterns of MAPT and APP isoform expression allowing in vitro modelling of ageing processes relevant for neurodegenerative disorders.

\section{Results}

\section{Distinct APP and Tau Isoform Regulation During Murine and Porcine Brain Development}

Human $A P P$ has 18 exons, of which exon 7 and 8 are subjects to alternative splicing. The longest isoform is APP770. Exclusion of exon 8 gives rise to APP751, whereas skipping of both exons results in the shortest APP695 variant (Fig. 1a). As for $M A P T$, exclusion of exon 2 and 3 gives rise to $0 \mathrm{~N}$ tau isoforms, whereas inclusion of exon 2 or exon 2 and 3 makes up the $1 \mathrm{~N}$ and $2 \mathrm{~N}$ isoforms, respectively. These three isoforms contain four (4R) or three (3R) microtubule-binding repeat domains depending on exon 10 inclusion or exclusion, respectively, and give rise to six main tau isoforms: 0N3R, $1 \mathrm{~N} 3 \mathrm{R}$, 2N3R and 0N4R, 1N4R, 2N4R (Fig. 1a). Corresponding exon organisation for mouse and porcine Mapt/MAPT and App/ $A P P$ were found using the Ensembl genome browser and are defined in Supplementary Table 1-3.

To determine isoform expression changes during embryonic development of the mouse and the pig brains, we used specimens representing foetal and adult cerebral cortex. We extracted RNA and protein from mice ranging in ages from embryonic day 10.5 (E10.5) to E18.5, postnatal day 14 (P14), P21, and from a 14-month-old adult mouse (Fig. 1b). Likewise, we extracted RNA and protein from E35 to E115 and from one 3-year-old adult pig (Fig. 1c). Species-specific RT-PCR primers were designed to detect APP/App and $M A P T /$ Mapt isoforms. During mouse cortical development from E10.5 to E18.5, we exclusively detected 0N3R tau isoform (Fig. 1b). At P14, a clear shift to 4R tau was observed while, at the same time, 3R tau had decreased to a low level. At this time point, faint bands emerged representing $1 \mathrm{~N}$ and $2 \mathrm{~N}$, but $0 \mathrm{~N}$ remained dominant as indicated by the corresponding strong band, and from P21, the 0N4R tau isoform was the predominant transcript variant detected (Fig. 1b). During porcine development from E40 to E100, we detected $1 \mathrm{~N} 3 \mathrm{R}$ and found that the porcine brain expressed $2 \mathrm{~N}$ and $1 \mathrm{~N}$ as well as $3 \mathrm{R}$ and $4 \mathrm{R}$ tau isoforms from E115 into adulthood (Fig. 1c). Immunoblotting using 3R- and 4R-specific antibodies (RD3 and RD4, respectively; Fig. 1a) confirmed the isoform switch from $0 \mathrm{~N} 3 \mathrm{R}$ to $0 \mathrm{~N} 4 \mathrm{R}$ in the mouse protein samples and the gradual switch from $1 \mathrm{~N} 3 \mathrm{R}$ to the four isoforms $1 \mathrm{~N} 3 \mathrm{R}, 1 \mathrm{~N} 4 \mathrm{R}, 2 \mathrm{~N} 3 \mathrm{R}$, and, $2 \mathrm{~N} 4 \mathrm{R}$ in the pig protein samples (Fig. 1d-f). Immunohistochemical staining of cortical sections showed that tau isoforms were expressed in neurons (MAP2+ cells) (Fig. 2, Supplemental Figure 1). With respect to $A P P / A p p$, the isoforms APP770 and APP751 were detected at low and decreasing levels while APP695 was the predominant isoform in both species throughout the time-course (Fig. 1b-c). As a reference, we detected six MAPT and three APP isoforms in the adult human cortex (Supplemental Figure 2A). 

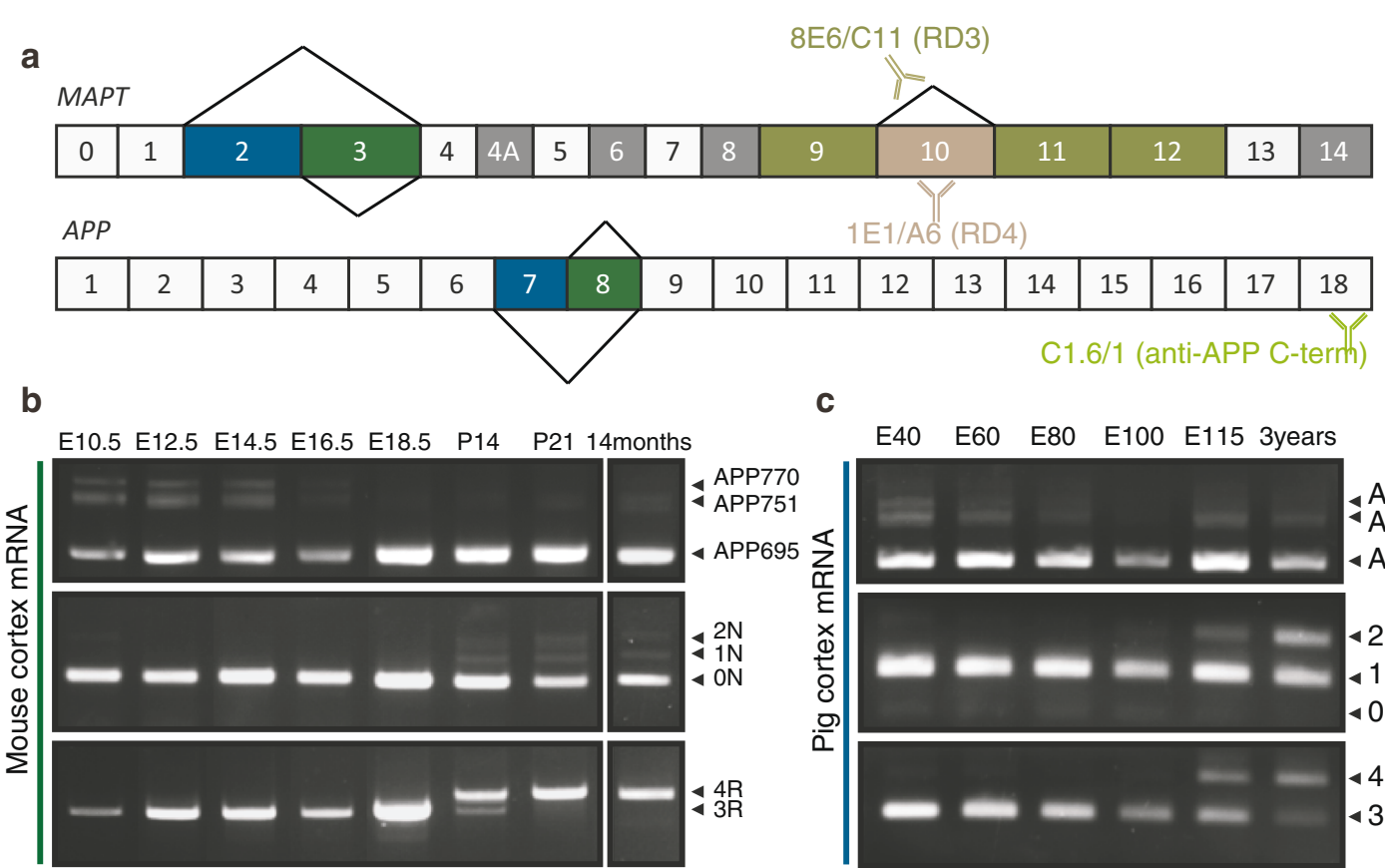

C
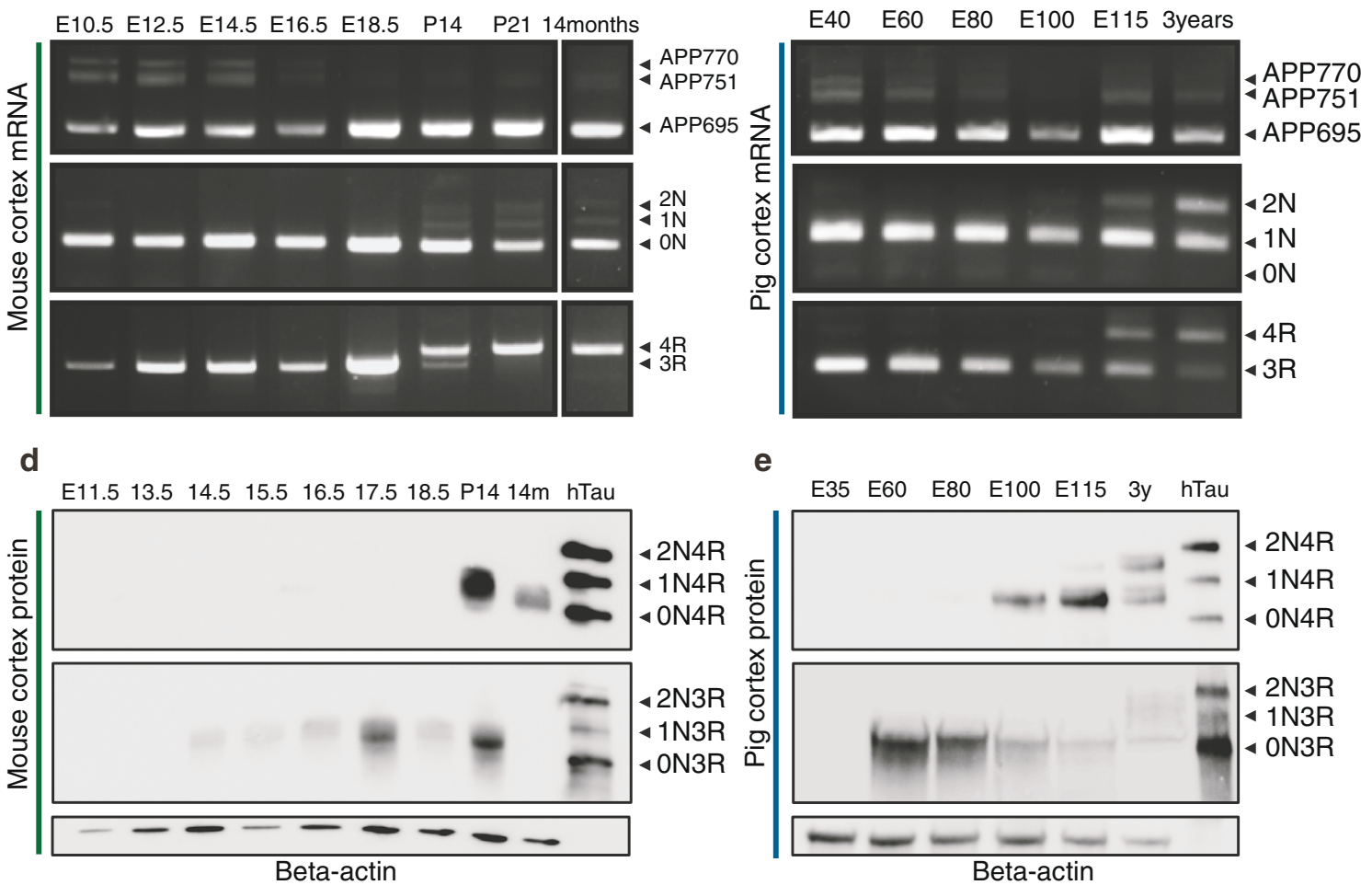

e
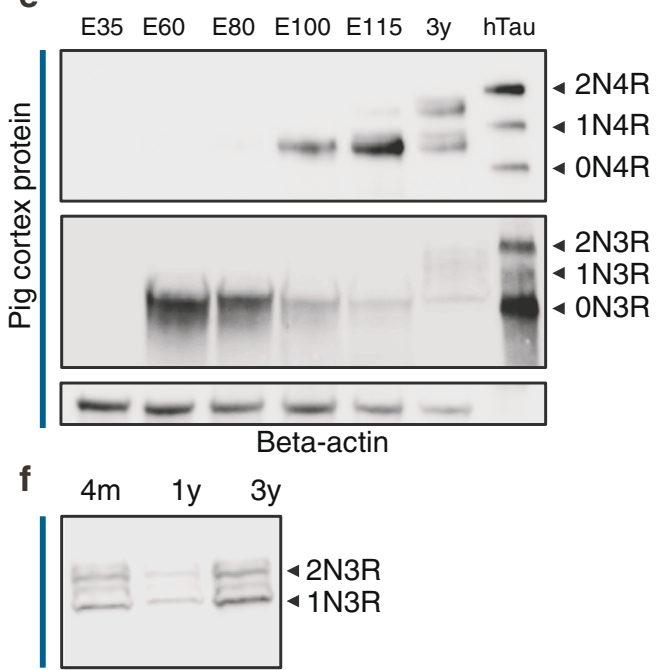

Fig. $1 A P P$ and $M A P T$ isoform expression during murine and porcine brain development. a Schematic illustration of MAPT and $A P P$ exon structure. b, c RT-PCR of App/APP and Mapt/MAPT isoform expression during embryonic development of cerebral cortex of the mouse (E11.5-E18.5; P14-P21; 14 months old) and the pig (E40-115; 3 years old) using species-specific RT-PCR primers. d, e Western blotting of protein samples equivalent to $\mathbf{b}$ and $\mathbf{c}$ using $3 \mathrm{R}$ (RD3; 8E6/C11) and

\section{Dynamic Isoform Expression Pattern of APP and Tau During Conversion of Porcine-Induced Neurons}

Porcine wild-type fibroblasts from a 4-month-old animal (PAF4M) were converted into induced neurons (iNs) by overexpression of mir-9/9*-124 and Ascll supported by small molecules as previously described [28](Fig. 3a-b). The starting fibroblast population stained positive for COL1A1 (Fig. 3c), a marker shown to be present in pig
4R (RD4; 1E1/A6) specific antibodies are recognising tau as illustrated in a. Recombinant human tau (hTau) protein marker used as a positive control. Beta-actin used as a loading control. f Western blotting of protein samples from adult pig cerebral cortex (4 months; 1 and 3 years old) using 3R (RD3; 8E6/C11) antibody. E, embryonic day/days postconception; $\mathrm{P}$, postnatal day

fibroblasts and absent in iNs [28]. Ten days post-transduction, the cells were immuno-positive for the pan-neuronal marker $\beta$-tubulin III (TUJ1) and mature neuronal marker MAP2 and with increased staining over time (Fig. 3d). At day 16 post-transduction, the cells became immunepositive for protein tau, which appeared located in soma and neurites at this time point (Fig. 3e).

To examine $A P P$ and $M A P T$ isoform expression changes during neuronal conversion, we first assessed gene expression 

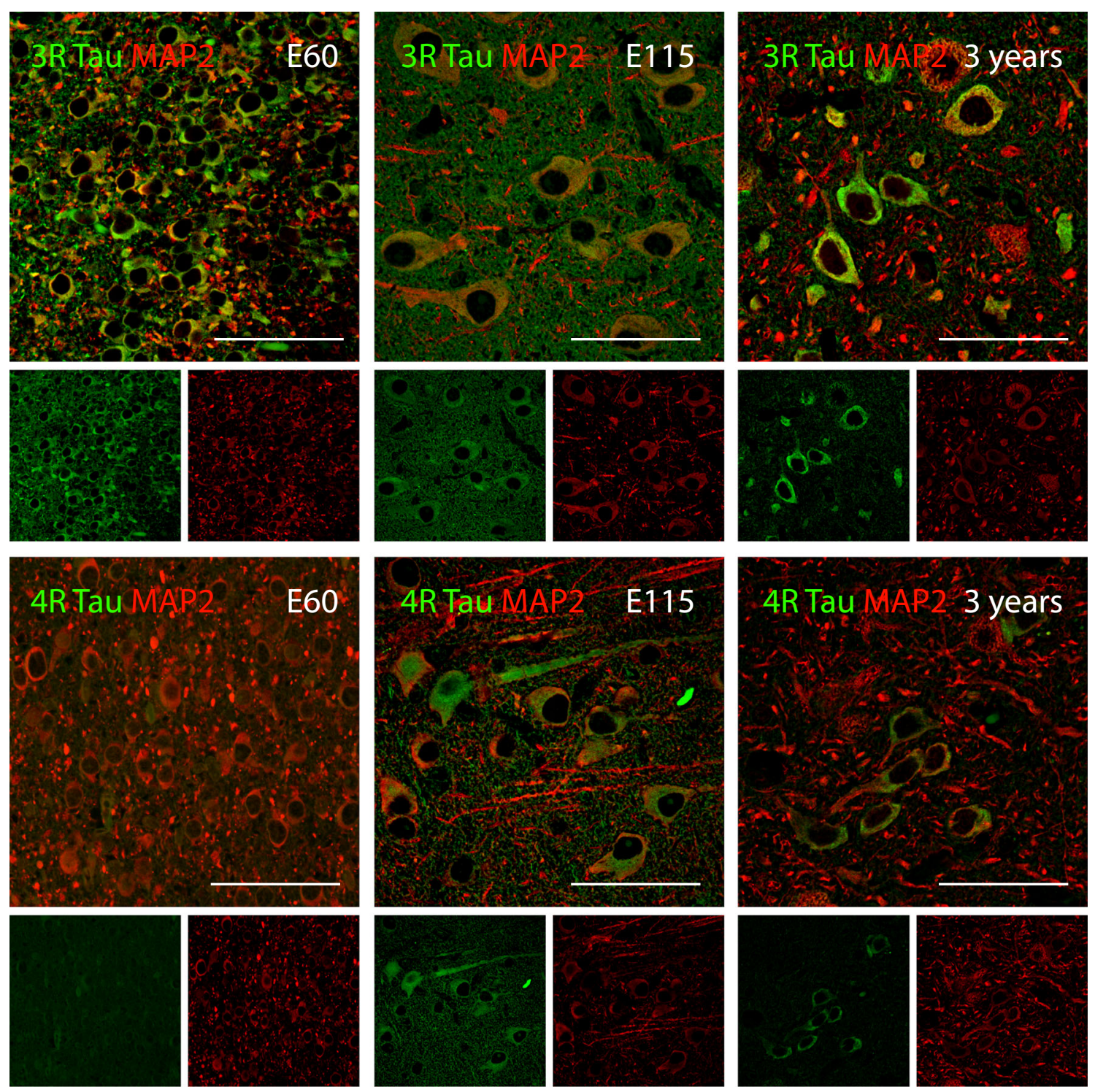

Fig. 2 MAPT cell-type specific isoform expression during porcine brain development. Immunohistochemical analysis of MAPT isoform expression in the cerebral cortex of the pig at different ages (E60; E115; 3 years old) using 3R (RD3; 8E6/C11) and 4R (RD4; 1E1/A6) specific

antibodies (green). Neurons identified using MAP2 specific antibody (red). E60 showed neuronal staining of 3R tau. The same was true for E115 and 3 years which, in addition, stained positive for $4 \mathrm{R}$ tau. Scale bars, $50 \mu \mathrm{m}$. E, embryonic day

levels of $A P P$ and $M A P T$ in fibroblasts and iNs at day 4, 10 and 28 using RNA sequencing (Fig. 3b, f). We found $A P P$ to be expressed at equal levels across samples, whereas the expression level of MAPT significantly increased as the cells converted into iNs (Fig. 3f). We next characterised the isoform composition of MAPT and APP using RT-PCR primers as in Fig. 1. This revealed dynamic alternative splicing events (Fig. $3 \mathrm{~g}$ ). While the gene expression level of APP did not change over time (Fig. 3f), the dominant transcript noticeably changed from APP770 in fibroblasts to APP695 in iNs (Fig. $3 \mathrm{~g})$. The shift in isoform expression occurred between day 4 and 10, at which time points both APP770 and APP751 were still detectable (Fig. 3g). MAPT expression was barely detectable in fibroblasts but increased to day 4 , where $2 \mathrm{~N}$, $1 \mathrm{~N}, 3 \mathrm{R}$ and $4 \mathrm{R}$ tau isoforms were clearly detected. This isoform composition did not change from day 4 to day 28 , although the relative amount of $1 \mathrm{~N}$ and $3 \mathrm{R}$ isoforms appeared to increase over time (Fig. 3g). DEXSeq analysis quantifying exon usage revealed significant differences in usage of $A P P$ exon 7 and 8 while the overall exon usage remained the same between fibroblasts and d28 iNs (Fig. 3i), which is in accord with the RT-PCR results, shown in Fig. 3g. Usage of all $M A P T$ exons was significantly increased in iNs compared to fibroblasts (Fig. 3j). The analysis showed that exon 2, 3 and 10 were expressed in the iNs, confirming the RT-PCR results (Fig. 3g). Immunostaining of iNs after 28 days of conversion 
a

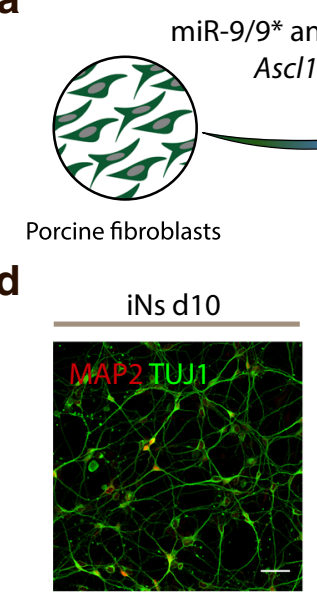

f

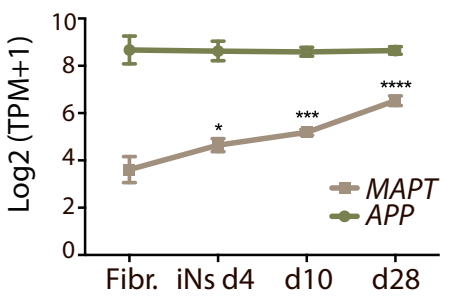

b

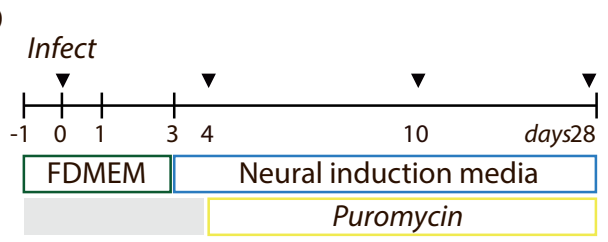

C

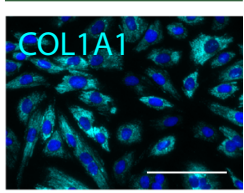

e

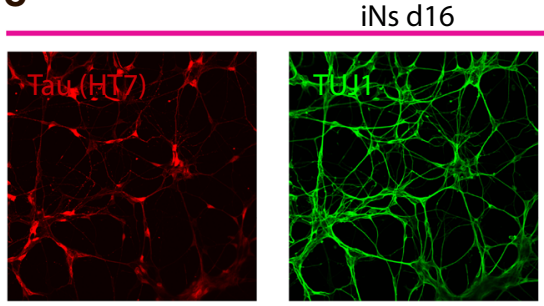

g

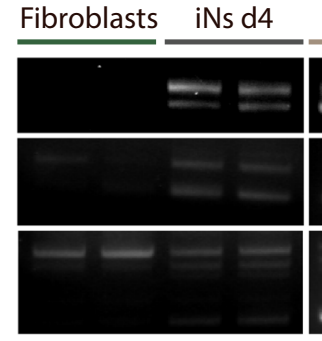

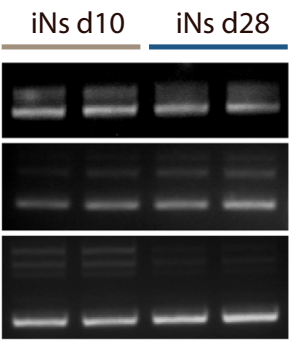

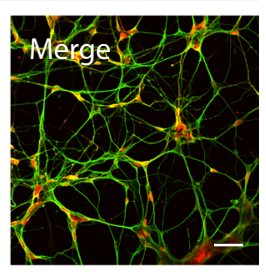

h i APP ENSSSCG00000012022

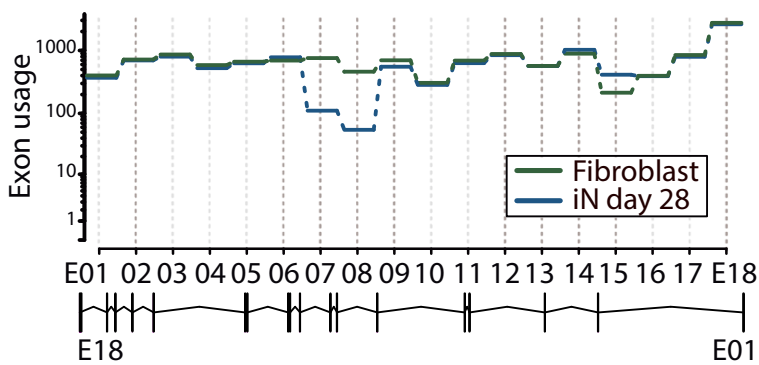

j MAPT ENSSSCG00000017311

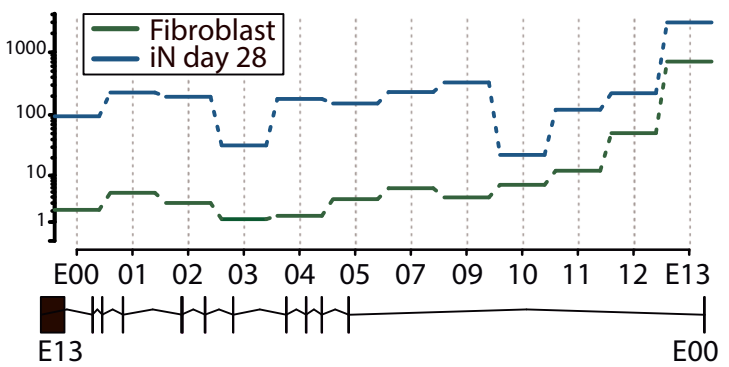

Fig. 3 Direct conversion of porcine fibroblasts into induced neurons. a, b Schematic illustration of conversion strategy. Wild-type porcine fibroblasts converted into induced neurons by overexpression of mir-9 and mir-124 (mir-9/9*-124) and Ascll cultured in neural induction media with puromycin selection up to day 28 after transduction. Arrows in $\mathbf{b}$ indicate time points for RNA extraction. $\mathbf{c}$ Immunostaining of fibroblasts for fibroblast marker COL1A1 (cyan). d Immunostaining of iNs for panneuronal TUJ1 (green) and mature marker MAP2 (red) after 10 and 28 days of conversion (d10, d28). e Immunostaining of iNs for TUJ1 (green) and tau (red, all isoforms) after 16 days of conversion (d16). All immunostainings counterstained with DAPI (blue). Scale bars, $50 \mu \mathrm{m}$. f $A P P$ and $M A P T$ expression level profiling $(\log 2(\mathrm{TPM}+1))$ during the conversion of fibroblasts into neurons at time points indicated in $\mathbf{b}$. Results presented as mean \pm s.d., $n=3$ for each time point. Two-way ANOVA followed by Dunnett's multiple comparisons test. g RT-PCR of $A P P$ and MAPT isoforms during neuronal conversion using speciesspecific RT-PCR primers. $n=2$ for each time point. h Quantification of junction read counts of $3 R$ and $4 R$ tau isoforms presented as $4 R / 3 R$ ratio of iNs 28 days post-transduction. Result presented as mean \pm s.d., $n=3$. (I, J) DEXSeq analysis quantifying differential $A P P$ and $M A P T$ exon usage between fibroblasts (green) and iNs 28 days after conversion (blue). Dotted lines in dark grey indicate differential exon usage. Ensembl IDs and exon structures for $A P P$ and $M A P T$ are shown. E, exon with $3 \mathrm{R}$ - and 4R-specific antibodies confirmed the isoform composition (Supplementary Figure 3). Exon junction counts revealed a $4 \mathrm{R} / 3 \mathrm{R}$ tau ratio of $17.34 \pm 7.11 \%$ (s.d.) (Fig. 3h). This encouraged us to look into the tau isoform composition of human-derived iNs. We utilised publicly available data on global gene expression of human iNs converted using $\mathrm{Ngn} 2$ and Ascll (N2AA) or shREST, mir-9/9*-124, Brn2 and Ascl1
(REST_sh-PBmPA) protocols [29, 30]. Visual inspection of the data with Integrative Genomics Viewer (IGV) revealed that all six MAPT isoforms were expressed in both datasets (Supplemental Figure 2B-E). Counting junction reads revealed a $4 \mathrm{R} / 3 \mathrm{R}$ tau ratio of $51.02 \pm 22.13 \%$ (s.d., $n=2$ ) for N2AA and $108.3 \pm 58.93 \%$ (s.d., $n=2$ ) for REST_sh-PBmPA converted iNs. 


\section{Differential Tau Isoform Composition in Induced Neurons from Embryonic and Adult Fibroblasts}

We next converted porcine fibroblasts derived from E35 embryos (PEF35D) and from 3-year-old adult animals (PAF3Y) into iNs (Fig. 4a) and evaluated the iNs at day 21 post-transduction. All fibroblasts in the starting population stained positive for COL1A1 (Fig. 4b). Conversion rates were assessed by staining for the presence of panneuronal markers $\beta$-tubulin III (TUJ1) and MAP2 (Fig. $4 \mathrm{c}-\mathrm{d})$. The protocol converted PEF35D, PAF4M and PAF3Y into iNs with similar rates, although significant lower MAP2 percentages were observed for PEF35D compared to adult fibroblast conversions (Fig. 4c). We then evaluated the isoform composition of $A P P$ and $M A P T$ in fibroblasts and iNs from PEF35D and PAF3Y (Fig. 4e). As in previous results, we found that $M A P T$ expression level increased during conversion from fibroblasts to iNs. In PEF35D-iNs, 1N3R tau isoform appeared to be the dominant MAPT transcript (Fig. 4e). By contrast, iNs derived from PAF3Y fibroblasts expressed similar tau isoforms as did PAF4M-iNs, as we found expression of $2 \mathrm{~N}, 1 \mathrm{~N}, 3 \mathrm{R}$ and $4 \mathrm{R}$ tau exons in these cells (compare Fig. $3 \mathrm{~g}$ and Fig. $4 \mathrm{e})$. Because we observed a tendency of adult fibroblasts to convert into iNs at higher rates than embryonic fibroblasts, we quantified and compared the expression level of $4 \mathrm{R}$ tau as a ratio between $4 R$ and $3 R$. This revealed a significantly lower 4R/3R ratio in PEF35D-iNs compared to PAF3YiNs (Fig. 4f). PAF3Y-iNs exhibited a $4 \mathrm{R} / 3 \mathrm{R}$ ratio of $20.1 \pm 0.96 \%$ (s.d.) similar to the tau ratio determined by junction counts of PAF4M-iNs (compare Fig. 3h and Fig. 4f). In addition, cell-type-specific but not age-dependent differences in $A P P$ isoform composition were detected (Fig. 4e). a
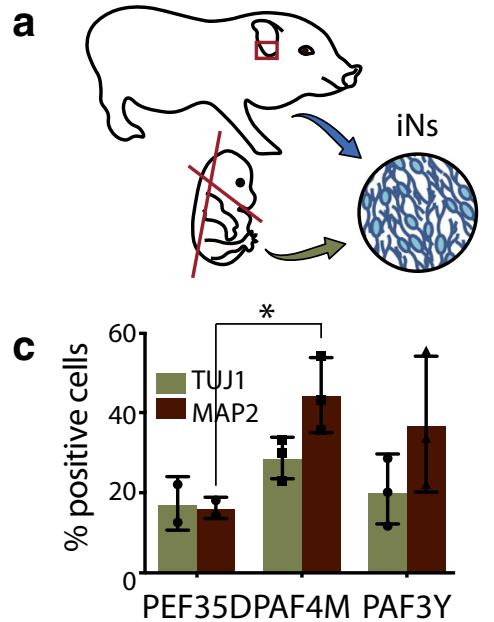

e
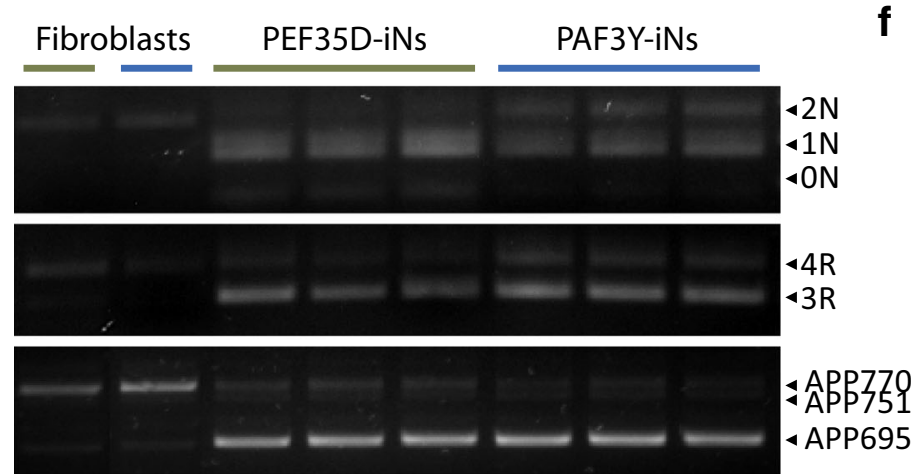

b

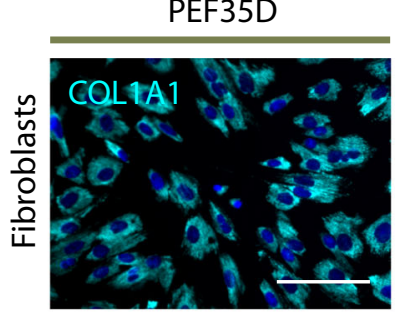

d

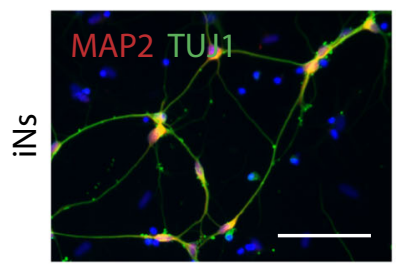

f
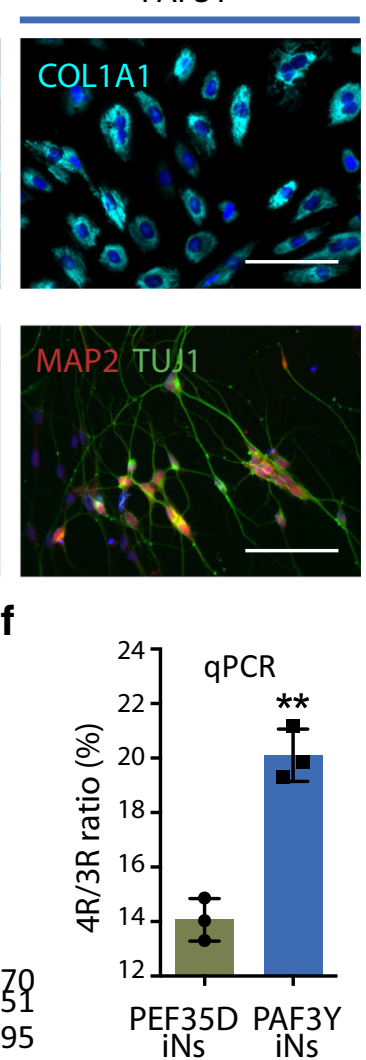

Fig. 4 Direct conversion of porcine embryonic fibroblasts into induced neurons. a Schematic illustration of direct conversion of porcine embryonic (E35) and adult (3 years) fibroblasts. b Immunostaining of embryonic (PEF35D, green) and adult (PAF3Y, blue) fibroblasts for fibroblast marker COL1A1 (cyan). c Quantification of the number of TUJ1 (green) and MAP2 (red) positive PEF35D $(n=2)$, PAF4M (porcine adult fibroblasts 4 months, $n=3)$ and PAF3Y $(n=3)$ fibroblast-derived induced neurons 21 days after conversion. Results presented as percentage $(\%)$, mean \pm s.d.. Two-way ANOVA followed by Sidak's multiple comparisons test. d Immunostaining of PEF35D- and PAF3Y-derived iNs after 21 days of conversion for TUJ1 (green) and MAP2 (red). All immunostainings counterstained with DAPI (blue). Scale bars, $50 \mu \mathrm{m}$. e RT-PCR of APP and MAPT isoform expression in PEF35D, PAF3Y and derived iNs using species-specific RT-PCR primers. $n=1$ for fibroblasts, $n=3$ for iNs. f RT quantitative PCR of $3 \mathrm{R}$ and $4 \mathrm{R}$ tau isoforms using exon junction-specific primers. Results presented as mean \pm s.d., $n=3$ for each sample. Unpaired $t$ test 


\section{Generation of Induced Neurons from Porcine Models of Alzheimer's Disease}

In order to demonstrate the applicability of iNs as a relevant disease model in neurodegenerative disorders, we generated and characterised iNs from fibroblasts from transgenic porcine models carrying AD-causing mutations in $A P P$ (Swedish mutation; APPsw) and/or PSEN1 (PSEN1M146I) (skin biopsies from 12 to 20 weeks old pigs) [31, 32]. Immunofluorescence of fibroblasts from wild-type (WT fibroblasts) and doubletransgenic APP/PS1 pigs (APP/PS1 fibroblasts) revealed distinct APP expression levels with the highest level in APP/PS1 fibroblasts due to overexpression of the human transgene $A P P 695 \mathrm{sw}$ in this model (compare Fig. 5a and b). The punctate staining of APP demonstrated a perinuclear and vesicular localisation indicative of correct synthesis and trafficking of the protein. Electrophoresis and immunoblotting using APP C-terminal antibody (Fig. 5c) did not detect any APP or APP derived fragments in WT or PS1 fibroblasts, indicating low levels of endogenous APP expression (Fig. 5d; lane WT-Ni and PS1-Ni). After treatment with $\gamma$-secretase inhibitor, $\alpha \mathrm{C}$ terminal fragment $(\alpha \mathrm{CTF})$, but not $\beta \mathrm{CTF}$ became detectable, demonstrating that very little endogenous APP was processed by $\beta$-secretase (Fig. 5d; lane WT-GSi and PS1-GSi; Fig. 5e). Overexpression of the APPSw transgene was evident in double-transgenic APP/PS1 fibroblasts as the accumulation of $\alpha$ CTFs were detected in non-inhibited cells (Fig. 5d; lane APP/PS1-Ni). Upon $\gamma$-secretase inhibition, a band representing $\beta \mathrm{CTF}$ was observed (Fig. 5d; lane APP/PS1GSi). Accordingly, the band of soluble APP (sAPP; consists of $\operatorname{sAPP} \alpha$ and $\operatorname{sAPP} \beta$ migrating as one band) decreased after treatment with both $\alpha$ - and $\beta$-secretase inhibitors (Fig. 5c, $\mathrm{f}, \mathrm{g})$. The molecular weight difference between the tg-sAPP band of the transgenic pig and the sAPP band of the WT pig represents the amino acid differences between transgenic APP695 and endogenous APP770.

Porcine iNs derived from PS1 and APP/PS1 fibroblasts displayed similar neuronal marker expression and conversion rates as those derived from WT fibroblasts (Fig. 5h-i). However, these iNs exhibited significantly fewer neurites per neuronal cell than WT-derived iNs (Fig. 5j). Neurite lengths did not differ (Fig. 5k).

We next assessed isoform composition of $A P P$ and MAPT in fibroblasts and iNs from WT, PS1 and APP/PS1 fibroblasts. Based on RT-PCR, we detected the expression of $2 \mathrm{~N}, 1 \mathrm{~N}, 3 \mathrm{R}$ and $4 \mathrm{R}$ tau in all iNs, but also a lower migrating band representing $0 \mathrm{~N}$ tau was visible (Fig. 51). We quantified and compared the 4R/3R tau ratio and found significantly higher $4 \mathrm{R} / 3 \mathrm{R}$ ratio in PS1-iNs and reduced 4R/3R ratio in APP/PS1iNs compared to WT-iNs (Fig. 5m). Using pig-specific APP primers, we confirmed that endogenous $A P P$ in all iN-lines changed from APP770 in fibroblasts to APP695 in iNs (Fig. 51). The transgenic APP contribution was found only in APP/
PS1 fibroblasts using primers detecting both human and porcine APP (Fig. 51).

We measured the levels of extracellular $A \beta 40$ and $A \beta 42$ during iN conversion of WT-, PS1- and APP/PS1-derived fibroblasts. The A $\beta$ levels were lower in WT and PS1 fibroblast than in iNs (data not shown). The A $\beta 42 / 40$ ratio was increased in iNs derived from PS1 and APP/PS1 fibroblasts compared to those of WT pigs (Fig. 5n).

\section{Discussion}

We identified $0 \mathrm{~N} 3 \mathrm{R}$ and $1 \mathrm{~N} 3 \mathrm{R}$ as the two main foetal tau isoforms present in the developing brains of mice and pigs, respectively. During human development, 0N3R tau is expressed [15] indicating that the presence of only $3 R$ tau isoforms is conserved during brain development of the three species. This is consistent with the need for neuronal plasticity during development as isoforms with 4 repeats (4R) bind and support microtubules more potently than $3 \mathrm{R}$ isoform. The $\mathrm{N}$ terminal parts of the isoform do not function in microtubule assembly [16, 33].

Differential splicing of tau between species was most evident in the adult brains. In the mouse adult brain, one tau isoform, 0N4R, was predominant. By contrast, four tau isoforms, $1 \mathrm{~N} 3 \mathrm{R}, 2 \mathrm{~N} 3 \mathrm{R}, 1 \mathrm{~N} 4 \mathrm{R}$ and $2 \mathrm{~N} 3 \mathrm{R}$, were found in the adult porcine brain, and this composition with $3 R$ and $4 R$ isoforms is more similar to the composition of six isoforms found in the human adult brain where $3 R$ and $4 R$ are expressed at similar levels (Goedert and Jakes, 1990). The time point at which longer $\mathrm{N}$-terminal isoforms started to be expressed was similar to the time point at which $4 \mathrm{R}$ isoforms could be detected for both species, suggesting that splicing of these domains is co-regulated.

The iPSC technology allows patient-specific neurons to be derived from somatic cells such as fibroblasts. The process of reprogramming involves rejuvenation as the resultant iPSCs resemble embryonic stem cells in regard to telomere length, mitochondrial metabolism and oxidative damage, which may not be useful for studying age-related pathologies. In addition, neurons derived from in vitro differentiation of iPSC or ESCs display developmental gene splicing as previously demonstrated for NCAM1 and MAPT [22, 23, 34]. In contrast, direct conversion of fibroblasts into induced neurons (iNs) circumvents the pluripotent intermediate state, and it has been demonstrated that iNs retain the transcriptomic and epigenetic age of the starting cells $[29,35]$. No authentic porcine embryonic stem cells or iPSCs are available [36, 37], but we recently established a protocol for directly converting porcine fibroblasts into iNs [28]. To our surprise, we found that iNs from adult porcine fibroblasts expressed the same four tau isoforms as do neurons of the adult pig brain. In publicly available RNAseq data, we found that also human fibroblast-derived 
a

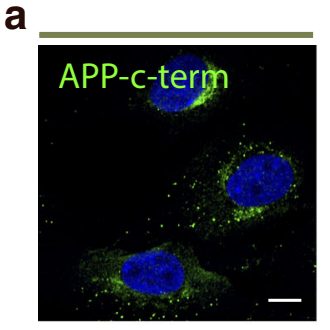

b

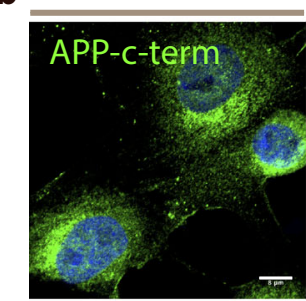

h

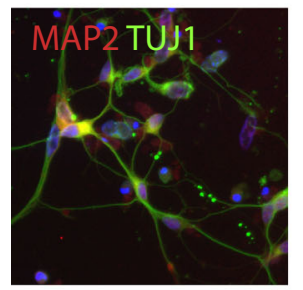

C

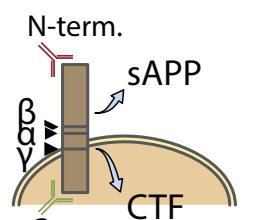

C-term. d

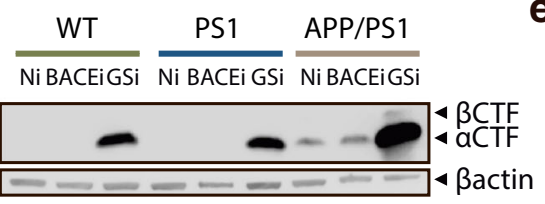

e

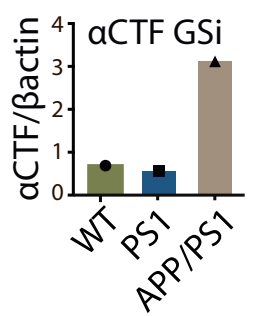

g

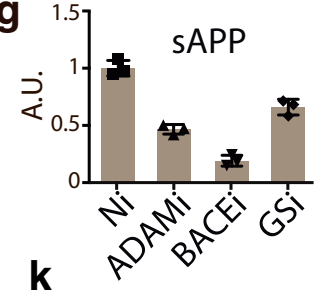

i

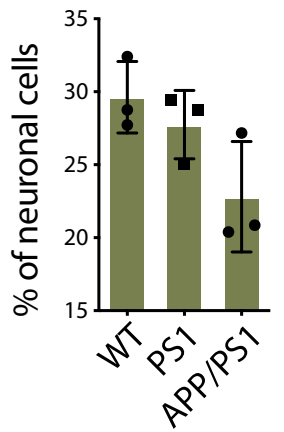

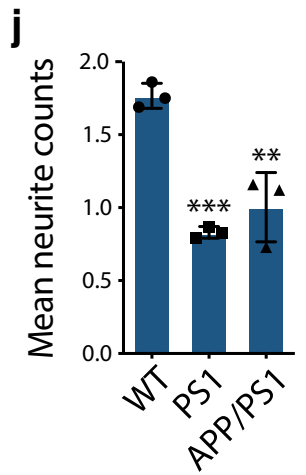

m

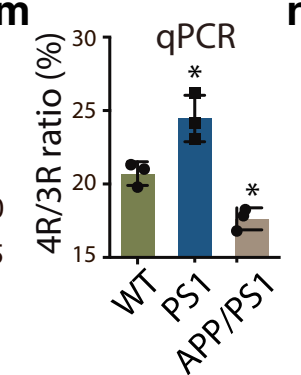

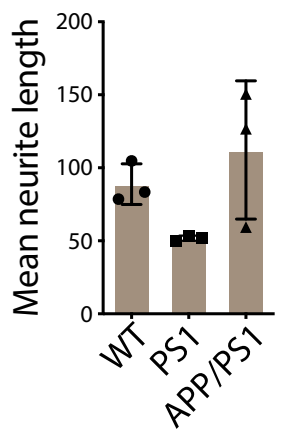

n

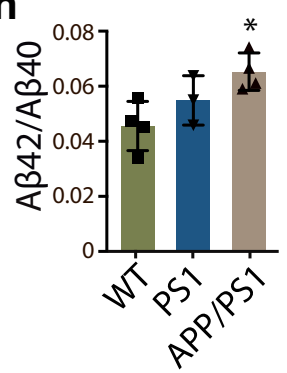

Fig. 5 Direct conversion of porcine fibroblast from transgenic models of Alzheimer's disease into induced neurons. a, b Immunostaining of fibroblasts from wild-type (WT) and double-transgenic APP/PS1 minipigs for APP $(\mathrm{Cl} 1 / 6.1$ antibody, green) recognising $\mathrm{C}$-terminal part of APP as illustrated in c. Counterstained with DAPI (blue). Scale bar, $8 \mu \mathrm{m}$. c Schematic illustration showing transmembrane localisation of APP, as well as cleavage sites of $\alpha-, \beta$ - and $\gamma$-secretases releasing sAPP and C-terminal fragments. Antibodies recognising N- (clone $22 \mathrm{C} 11$ ) and $\mathrm{C}$ - (clone $\mathrm{C} 1 / 6.1$ ) terminal parts of APP are shown in red and green, respectively. d Western blot of APP processing in fibroblast lysates from WT, single- (PS1) and double- (APP/PS1) transgenic minipigs with or without $(\mathrm{Ni}) \gamma-(\mathrm{GSi}$; L685,458; $1 \mu \mathrm{M})$ or $\beta$ (BACEi; $\beta$-secretase IV; $10 \mu \mathrm{M}$ ) secretase inhibition using anti-APP $\mathrm{C} 1 / 6.1$ antibody. $\beta$-actin used as a loading control. e Quantification of $\alpha \mathrm{CTF}$ bands in (D). $n=1$ for each sample. $\mathbf{f}$ Western blot of sAPPconditioned media from triplicates of WT and double-transgenic minipig with or without $\alpha$ - (ADAMi; TAPI-1; $10 \mu \mathrm{M}), \beta$ - (BACEi; $\beta$ secretase IV; $10 \mu \mathrm{M})$ and $\gamma-(\mathrm{GSi} ; \mathrm{L} 685,458 ; 1 \mu \mathrm{M})$ secretase inhibition

iNs expressed the same tau isoforms as the adult human brain. To our knowledge, this observation regarding iNs has never been reported before, and we hypothesised that tau isoform using anti-APP antibody 22C11. g Quantification of transgenic (tg) sAPP in f. Results presented as mean \pm s.d., $n=3$ for each sample. $\mathbf{h}$ Representative immunostaining of transgenic pig-derived iNs for panneuronal TUJ1 (green) and mature marker MAP2 (red). Counterstained with DAPI (blue). i, j, k Quantification of neuronal cells (TAU+/DAPI), mean neurite counts and mean neurite length. Results presented as mean \pm s.d., $n=3$ for each sample. One-way ANOVA followed by Dunnett's multiple comparisons test. I RT-PCR of APP and MAPT isoform expression in WT-, PS1- and APP/PS1-derived iNs using speciesspecific RT-PCR primers. $n=1$ for fibroblasts, $n=3$ for iNs. m RT quantitative PCR of $3 \mathrm{R}$ and $4 \mathrm{R}$ tau isoforms using exon junctionspecific primers. Results presented as mean \pm s.d., $n=3$ for each sample. One-way ANOVA followed by Dunnett's multiple comparisons test. $\mathbf{n}$ ELISA measurements of $\mathrm{A} \beta-40$ and -42 in media of WT, PS1 and APP/PS1 fibroblasts and iNs after 28 days of conversion. Results presented as mean \pm s.d., $n=4$ (WT; APP/PS1) or 3 (PS1) for each sample. One-way ANOVA followed by Dunnett's multiple comparisons test

composition could be used as a marker of cellular age [25]. To this end, we investigated tau isoforms in porcine embryonic fibroblasts converted into iNs and found that $1 \mathrm{~N} 3 \mathrm{R}$, the 
porcine foetal isoform of tau, was the dominant transcript in these cells. We detected only low levels of 4R tau compared to levels observed in adult porcine-derived iNs.

The human and porcine iNs were converted using different transcription factor combinations, suggesting that the induction of 4R tau isoforms is not related to the choice of direct conversion strategy [25]. These experiments were all based on bulk RNA extractions, and isoform expression analysis remains to be performed at a single-cell level. The $3 \mathrm{R}$ and $4 \mathrm{R}$ tau antibodies used in this study were both mouse immunoglobulins and, therefore, could not be used together to answer this question.

Tau participates in axonal transport of neurons, and it was recently shown that imbalance in the normal $3 \mathrm{R} / 4 \mathrm{R}$ ratio could impair transportation of APP [38], which highlights the importance of correct tau isoform expression to model certain aspects of neurodegeneration. We found cell-typespecific isoform expression of $A P P$ during conversion from fibroblasts to neurons, i.e. switch of $A P P 770$ to the neuronal isoform APP 695 between day 4 and 10 in the conversion process without changing the general expression level of $A P P$. Similarly, endogenous APP mRNA in our single- and double-transgenic animals to iNs was spliced normally, and quantification of $A \beta$ detected an increase in the $A \beta 42 / 40$ ratio in transgenic pigs consistent with the effects of PSEN1M146I and APPSw transgenes. This means that the first crucial steps in $\mathrm{AD}$ pathogenesis are faithfully modelled in these iNs.

The finding that the tau isoform composition in porcine and human-induced neurons resembles that of the adult brain of each species has implications for the choice of the cellular system to use for in vitro modelling and drug screening purposes of age-related neurodegenerative disorders such as Alzheimer's disease.

\section{Materials and Methods}

\section{Tissue}

Porcine foetuses were obtained from artificially inseminated landrace sows (Sus scrofa). Pregnant sows were anaesthetised by inhalation of $35-70 \% \mathrm{CO}_{2}$ for $1 \mathrm{~min}$ and sacrificed by exsanguination. The uteri were recovered and the foetuses from 35/40, 60, 80, 100 and 115 days post-conception were quickly removed, dissected and flash-frozen in liquid nitrogen. Tissue for immunohistochemical analysis was immersed in neutral-buffered $10 \%$ formalin (Sigma-Aldrich).

Murine foetuses were obtained from timed mating of mice acquired from Taconic M\&B, Ry, Denmark. Females were examined the following morning, and the stage of development was designated embryonic day 0.5 (E0.5). Pregnant mice were killed by cervical dislocation. Foetuses were dissected from the age of embryonic day 10.5, 11.5, 12.5, 13.5,
$14.5,15.5,16.5,17.5$ and 18.5 (day of birth), and postnatal day 14 (P14) and P21. The anterior part of the embryo was collected from E10.5 to E16.5, and the brains were dissected from E17.5, E18.5, P14, P21 and from a 14-months-old adult mouse. Samples were immediately flash-frozen in liquid nitrogen. Tissue for immunohistochemical analysis was immersed in neutral-buffered 10\% formalin (Sigma-Aldrich).

\section{Cell Culture}

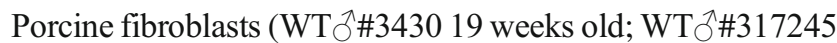
3 years; PS1 $\$$ \#4902 12 weeks; APP/PS1 \$\#6036 20 weeks old) were obtained from ear biopsies from Göttingen minipigs and cultured in Dulbecco's Modified Eagle's Medium (DMEM; Sigma-Aldrich), 1\% FBS (Life Technologies), 1\% $\mathrm{P} / \mathrm{S}(\mathrm{P} / \mathrm{S}, 10,000$ units $/ \mathrm{mL}$ and $10,000 \mu \mathrm{g} / \mathrm{mL}$; Life Technologies), $1 \%$ Gln $(2.92 \mathrm{~g} / 100 \mathrm{~mL})$ and $6.667 \mathrm{ng} / \mathrm{mL}$ fibroblast growth factor-basic (bFGF; Life Technologies) in $5 \% \mathrm{CO}_{2}$ in a humidified chamber at $37^{\circ} \mathrm{C}$. The outgrowing fibroblasts were washed with phosphate buffered saline (PBS; Life Technologies) and harvested by trypsinisation for 2 3 min at $37{ }^{\circ} \mathrm{C}$ (0.05\% trypsin EDTA; Life Technologies) and cultured for one additional passage before long-term storage at $-135^{\circ} \mathrm{C}$. Established fibroblasts were cultured in fibroblasts media and maintained in $5 \% \mathrm{CO}_{2}$ in a humidified chamber at $37{ }^{\circ} \mathrm{C}$ (unless otherwise stated) and split in a 1:3 ratio every $2-3$ days using $0.05 \%$ trypsin EDTA (Life Technologies).

Porcine embryonic fibroblasts were obtained from Göttingen minipig embryos at day 35 of gestation. The heads of the foetuses were removed together with the remaining internal organs, and the bodies were fragmented and incubated with $0.05 \%$ trypsin EDTA (Life Technologies) containing DNAse (1:50; Roche) in $37^{\circ} \mathrm{C}$ for $5-10 \mathrm{~min}$ and disaggregated by pipetting using a $21 \mathrm{G}$ needle (Terumo). Embryonic fibroblasts were maintained as described for porcine adult fibroblasts. All fibroblasts used in this study tested negative for mycoplasma (MYCOPLASMACHECK, Eurofins Genomics). Göttingen minipigs are housed at Research Center Foulum, Department of Animal Science, Aarhus University.

\section{Lentivirus Production}

For direct fibroblast-to-neuron conversion, we used commercially available plasmids pTight-9-124, Tet-O-Ascl1 and FUW-M2rtTA (Addgene plasmid \#31874, \#27150 and \#20342). Lentiviral particles were produced using 2nd generation lentiviral packaging vectors (psPAX2 and pMD2.G, Addgene plasmid \#12260 and \#12259). In brief, HEK293T/ 17 (ATCC, cat. no. CRL-11268) cells were transfected with the plasmid of interest together with packaging and envelope plasmids using Lipofectamine ${ }^{\mathrm{TM}} 3000$ Transfection Reagent 
(Life Technologies). The supernatant containing the virus particles was collected 24 and $48 \mathrm{~h}$ post-transfection, filtered $(0.45 \mu \mathrm{m}$ filter, Frisenette), concentrated by ultracentrifugation $22500 \mathrm{~g}$ for $1 \mathrm{~h}$ and $30 \mathrm{~min}$ at $4{ }^{\circ} \mathrm{C}$ and resuspended in PBS and snap-frozen and stored at $-80^{\circ} \mathrm{C}$. Lentiviral titration was performed by quantitative PCR using 7500 Fast real-time PCR system (Applied Biosystems) and primers against the lentiviral backbone (LV2) and albumin $(A L B)$ as an internal control for normalisation. Titre was calculated by comparing integrated viral DNA content against a vector reference, pWPXL (pWPXL was a gift from Didier Trono) with known titre determined by green fluorescent protein (GFP) expression in transduced cells. All viruses had titre above $1.0 \times 10^{8}$.

\section{Direct Reprogramming}

The direct conversion was performed as previously described [28]. In brief, porcine fibroblasts were plated onto poly-Lornithine hydrobromide $(0.1 \mathrm{mg} / \mathrm{mL}$; Sigma-Aldrich)-, laminin L2020 (10 $\mu \mathrm{g} / \mathrm{mL}$; Sigma-Aldrich)- and fibronectin (10 $\mu \mathrm{g} / \mathrm{mL}$; Sigma-Aldrich)-coated cell culture-treated plastic plates (Fisher Scientific) or acid-treated coverslips (VWR). Fibroblasts were transduced with concentrated lentivirus at the multiplicity of infection 20 of individual vectors. After $16 \mathrm{~h}$, the media was changed and supplemented with doxycycline (DOX; $2 \mu \mathrm{g} / \mathrm{mL}$; Sigma-Aldrich). On day 3 post-transduction, the media was replaced by neuronal conversion media (Neurobasal media (Life Technologies) and DMEM/F12 (Life Technologies) mixed at a 1:1 ratio containing $1 \%$ glucose (30\%), 1\% P/S (10,000 units/mL and 10,000 $\mu \mathrm{g} / \mathrm{mL}$; Life Technologies), $1 \%$ Glutamax (Life Technologies), $1 \%$ ITS-A (Life Technologies), 1\% B27 (Life Technologies) and $1 \%$ N2 (Life Technologies)) supplemented with the small molecules CHIR99021 (2 $\mu \mathrm{M}$; Stemgent), SB-431542 (10 $\mu \mathrm{M}$; Tocris), LDN-193189 (0.5 $\mu \mathrm{M}$; Stemgent), A-83 $(0.5 \mu \mathrm{M}$; Tocris), Forskolin (5 $\mu \mathrm{M}$; Sigma-Aldrich) and growth factors LM-22A4 ( $2 \mu \mathrm{M}$; Tocris), GDNF (2 ng/mL; R\&D Systems), NT3 (10 ng/mL; Peprotech) and db-cAMP ( $0.5 \mathrm{mM}$; Sigma-Aldrich), as well as the epigenetics regulator valproic acid (VPA; $1 \mathrm{mM}$; Sigma-Aldrich) and antibiotics (puromycin; $1 \mu \mathrm{g} / \mathrm{mL}$; Gibco). Fibroblasts derived from transgenic animals were puromycin-resistant [31] and all conversions comparing WT to transgenic fibroblast were therefore carried out without puromycin selection but with the addition of AraC ( $2 \mu \mathrm{M}$; Sigma-Aldrich) to inhibit proliferation of non-converting fibroblasts. Half media changes were performed every 2-3 days until analysis.

\section{Immunocytochemistry}

Cells plated on coverslips (VWR) were washed twice with PBS (Life Technologies) and fixated in 4\% paraformaldehyde (PFA; Santa Cruz Biotechnologies, Inc.) for $15 \mathrm{~min}$ at $4{ }^{\circ} \mathrm{C}$, then washed with PBS (Life Technologies) and distilled water (Life Technologies) and left to dry for $10 \mathrm{~min}$. The cells were then permeabilised for $10 \mathrm{~min}$ at RT in PBS (Life Technologies) containing $0.25 \%$ Triton X (Sigma-Aldrich) (PBT) and blocked for $1 \mathrm{~h}$ at RT in PBT supplemented with 5\% donkey serum (Almeco). The primary antibodies were diluted in blocking solution and cells and antibodies were incubated $\mathrm{O} / \mathrm{N}$ at $4{ }^{\circ} \mathrm{C}$ : rabbit anti-beta tubulin III (1:1000; Biolegend; 802,001), chicken anti-MAP2 (1:2500; Abcam; ab92434), mouse anti-TAU (1:500, Thermo Scientific; clone HT7 MN1000), mouse antiAPP-C-terminus (C1/6.1; 1:2000; Biolegend), mouse anti-Tau 3-repeat isoform RD3 (1:1000; Sigma-Aldrich; 8E6/C11), mouse anti-Tau 4-repeat isoform RD4 (1:500; Sigma-Aldrich; 1E1/A6). Thereafter, cells were washed with PBT and blocked for $10 \mathrm{~min}$. Secondary antibodies conjugated to fluorophores 647 or -488 (Invitrogen; A-31571, Jackson Immunoresearch; 703-545-155, Invitrogen; A10042) were diluted in blocking buffer and applied for $1 \mathrm{~h}$ at RT, then washed and counterstained with 49,6-diamidino-2-phenylindole (DAPI; $1 \mu \mathrm{g} / \mathrm{mL}$; Sigma) diluted in PBS (Life Technologies). Cells were washed three times with PBS (Life Technologies) and mounted using PVADABCO (Sigma-Aldrich).

\section{Immunofluorescence Staining on Tissue Sections}

Formalin-fixated tissues were embedded in paraffin blocks and cut in 2-4- $\mu \mathrm{m}$ sections and mounted on superfrost plus slides. Sections were then deparaffinised in xylene $\mathrm{O} / \mathrm{N}$ and rehydrated (30 min 99\% EtOH; 20 min 96\% EtOH; 10 min 70\% EtOH; rinsed with distilled water). Antigens were retrieved by boiling in TEG buffer (10 mM Tris, $0.5 \mathrm{mM}$ EGTA, $\mathrm{pH}$ 9) and aldehyde groups were shielded in $50 \mathrm{mM} \mathrm{NH}_{4} \mathrm{Cl}$ in PBS. Sections were rinsed $30 \mathrm{~min}$ in blocking solution $1 \% \mathrm{BSA}, 0.2 \%$ gelatin in PBS. The primary antibodies were diluted in $0.1 \%$ BSA, $0.3 \%$ Triton $\mathrm{X}-100$ in PBS and incubated $\mathrm{O} / \mathrm{N}$ in a humidity chamber at $4{ }^{\circ} \mathrm{C}$ : mouse anti-Tau 3-repeat isoform $\mathrm{RD} 3(1: 100$; Sigma-Aldrich; 8E6/C11), mouse anti-Tau 4-repeat isoform RD4 (1:100; Sigma-Aldrich; 1E1/A6). The following day, the sections were rinsed three times and incubated for $30 \mathrm{~min}$ at room temperature with secondary antibodies conjugated to fluorophores -647 or -488 (Jackson Immunoresearch) followed by three washes in PBS and mounting of coverslips (VWR) using PVA-DABCO (Sigma-Aldrich).

\section{Microscopy}

Quantification of the percentage of TUJ1 and MAP2-positiveinduced neurons were obtained using Cellomics Array Scan (Array Scan VTI, Thermo Fischer) by applying the program 'Target Activation' and 'Neuronal Profiling'. Objects were selected based on the intensity and size parameters set by the user. Images were obtained with a Zeiss LSM confocal 1 sm780 or Leica fluorescent microscope (Wetzlar, Germany). 


\section{Protein Extraction and Western Blotting}

Cells and tissues were lysed in RIPA lysis buffer (prepared in house; $50 \mathrm{mM}$ Tris $\mathrm{HCl}, 150 \mathrm{mM} \mathrm{NaCl} 150 \mathrm{mM}$, Sodiumdeoxycholate $0.5 \%, 0.1 \%$ SDS, $1 \%$ NP40) containing protease inhibitors (c0mplete Mini, EDTA-free protease inhibitor cocktail; Roche) followed by sonication (Bioruptor®). Tissue samples were homogenised in RIPA buffer using a tissue-grinder before sonication. Homogenates were centrifuged at $5000 \mathrm{~g}$ for $10 \mathrm{~min}$ at $4{ }^{\circ} \mathrm{C}$. The supernatant was collected, and protein concentration was measured using Bradford protein assay (Bio-Rad) following the manufacturer's protocol.

Proteins were separated by SDS-PAGE on $16.5 \%$ Tris/ Tricine gels (Bio-Rad) for cell lysates, 7.5\% Glycine gels (Bio-Rad) for conditioned media and 10\% Glycine gels (Bio-Rad) for tissue lysates and were transferred to nitrocellulose or PVDF membranes, respectively $(0.2 \mu \mathrm{m}$; Bio-Rad). The membranes were boiled in PBS (Life Technologies) for 5 min to unmask the epitopes and blocked in 5\% skim milk (5 g Skim Milk (Difco ${ }^{\mathrm{TM}}$ ), $50 \mathrm{~mL}$ TBS-T containing $100 \mathrm{~mL}$ 10x Tris Buffered Saline (Fisher BioReagents), $900 \mathrm{~mL}$ water, $2 \mathrm{~mL} \mathrm{100 \%} \mathrm{Tween} 20$ (Sigma-Aldrich)) for $1 \mathrm{~h}$ prior to probing with primary antibody $\mathrm{O} / \mathrm{N}$ : mouse anti-APP-C-terminus (C1/6.1; 1:2000; Biolegend), rabbit anti-APP-N-terminus (22C11; 1:2000; Calbiochem; MAB348), anti-beta-actin (1:10,000; Abcam; ab6276), mouse anti-Tau 3-repeat isoform RD3 (1:10,000; Sigma-Aldrich; 8E6/C11) and mouse antiTau 4-repeat isoform RD4 (1:2000; Sigma-Aldrich; 1E1/ A6). Membranes were washed $4 \times 30 \mathrm{~min}$ and applied with horse-radish peroxidase-conjugated secondary antibodies, Goat anti-mouse (1:2000; Dako) or Goat anti-rabbit (1:2000; Dako) for $1.5 \mathrm{~h}$ followed by additional $4 \times 30 \mathrm{~min}$ of washing. Blots were developed using Clarity ${ }^{\mathrm{TM}}$ Enhanced Chemiluminescence (Bio-rad) by a film based (Konica Minolta) or digital imaging. For reprobing, membranes were stripped using Re-Blot Strong Solution (Calbiochem).

\section{ELISA}

ELISA was performed on conditioned media using ELISA A $31-40$ and 1-42 kits (Thermo Fisher Scientific) following the manufacturer's protocol. The medium was harvested from three experiments at day 28 post-transduction, and $1 \mathrm{mM}$ PMSF was added prior to storage at $-20{ }^{\circ} \mathrm{C}$ until analysis.

\section{RNA Extraction and CDNA Synthesis}

RNA from fibroblasts and iNs were extracted using an automated RNA purification system, Maxwell ${ }^{\circledR}$ RSC simplyRNA Tissue Kit (Promega). Murine and porcine cerebral cortical tissues were homogenised in TriReagent (Sigma-Aldrich) by an RNAse-free tissue-grinder. RNA was extracted using chloroform and isopropanol precipitation. RNA concentration and purity were evaluated using NanoDrop 1000 version 3.7.1. (Thermo Fisher Scientific). Complementary DNA (cDNA) was synthesised from RNA using iScript ${ }^{\mathrm{TM}} \mathrm{cDNA}$ Synthesis Kit (Bio-Rad) with a 1:1 mix of oligo(dT) and random hexamer primers.

\section{RT-PCR and RT-qPCR}

RT-PCR was carried out using Q5 high-fidelity or OneTaq Hotstart polymerases (New England Biolabs) according to the manufacturer's protocol. PCR products were run on a $2 \%$ agarose gel. RT-qPCR was performed on pre-diluted cDNA and mixed with LightCycler® 480 SYBR Green I Master (Roche). Relative gene expression was calculated by a standard curve method [39] using serial dilutions of a pool of cDNA from all samples present in the study. Expression levels were normalised to the geometric mean of GAPDH and HPRT1 reference genes. Negative controls as cDNA synthesis without reverse transcriptase and water only samples were included. All runs were performed in triplicates. Primers used for RT-PCR and RT-qPCR are shown in Supplementary Table 4.

\section{RNA Sequencing and Bioinformatics}

Raw sequencing reads from published RNA-sequencing (RNAseq) experiments were extracted as FASTQ from National Center for Biotechnology Information Short Read Archive using Galaxy (Version 2.8.1.3) [40].

For pig RNAseq data files (GSE146494), reads were trimmed using Trimmomatic [41] to remove the first 10 bases from the start of the read. Reads were aligned to the porcine genome build Sscrofa11.1 (Ensemble release 98) using HISAT2 aligner (v2.1.0) [42]. Transcript quantification and junction counts were generated from featureCounts (v1.6.3) [43] and the read counts were normalised for effective gene length, and sequencing depth to yield Transcripts Per Kilobase Million (TPM). The raw junction counts were normalised for sequencing depth. We extracted counts of MAPT (ENSSSCG00000017311) junctions exon 9-10 ( $\mathrm{X}_{9,10}$, chr12, position $17,122,167$ to $17,112,299), 9-11\left(\mathrm{X}_{9,11}\right.$, chr12, position $17,122,167$ to $17,108,761$ ) and $10-11$ ( $\mathrm{X}_{10,11}$, chr12, position $17,112,207$ to $\left.17,108,761\right)$. The $3 \mathrm{R}$ splice form is represented by $\mathrm{X}_{9,11}$ and $4 \mathrm{R}$ by both $\mathrm{X}_{9,10}$ and $\mathrm{X}_{10,11}$. The percentage of MAPT $4 \mathrm{R}$ usage was subsequently calculated by the following: $100 *\left(0.5 *\left(\mathrm{X}_{9,10}+\mathrm{X}_{10,11}\right)\right) /$ $\left(\mathrm{X}_{9,11}\right)$. The number of reads per exon in MAPT and $A P P$ genes was counted using DEXSeq-Count (v1.28.1.0), and differential exon usage was determined using DEXSeq (v1.28.1) tool [44]. Differentially expressed genes were determined from count tables using DEseq2 (v2.11.40.2) [45]. Disease and biofunction pathway analysis was performed using the QIAGEN Ingenuity Pathway Analysis software. 
For human RNAseq data (E-MTAB-3037; GSE132154), reads were aligned to the human genome build hg19/GRCh38 (Ensemble release 94) using HISAT2 aligner (v2.1.0) [42]. Reads aligned to MAPT (ENSG00000186868, chr17, position $45,894,382$ to $46,028,334$ ) were visually inspected using IGV software. Junction counts were generated from featureCounts (v1.6.3) [43]. The raw junction counts were normalised for sequencing depth. We extracted counts of MAPT (ENSG00000186868) junctions exon 9-10 $\left(\mathrm{X}_{9,10}\right.$, chr17, position 45,996,664 to 46,010,310), 9-11 ( $\mathrm{X}_{9,11}, \mathrm{chr17}$, position $45,996,664$ to $46,014,243)$ and $10-11\left(\mathrm{X}_{10,11}, \mathrm{chr} 17\right.$, position $46,010,402$ to $46,014,243)$. The $3 \mathrm{R}$ splice form is represented by $X_{9,11}$ and $4 R$ by both $X_{9,10}$ and $X_{10,11}$. The percentage of $M A P T$ 4R usage was subsequently calculated by the following: $100 *\left(0.5 *\left(\mathrm{X}_{9,10}+\mathrm{X}_{10,11}\right)\right) /\left(\mathrm{X}_{9,11}\right)$.

Supplementary Information The online version contains supplementary material available at https://doi.org/10.1007/s12035-020-02258-w.

Acknowledgements We thank Malin Parmar and Shelby Shrigley for contributing reagents and help with high content screening.

Author Contributions $\mathrm{MH}$ performed the experiments. MH, MD, PQ, IEH and ALJ designed and analysed the experiments. MH wrote the original manuscript. All authors reviewed, edited and approved the final manuscript.

Funding MH is supported by a fellowship from the Graduate School of Health, Aarhus University. MD was supported by Lundbeckfonden grant no. DANDRITE- R248-2016-2518.

Data Availability The GEO accession number for RNAseq reported in this paper is GSE146494.

\section{Compliance with Ethical Standards}

Competing Interests The authors declare that they have no conflicts of interest.

Ethics Approval All experiments involving the animals are reviewed and approved by the Danish Animal Experiment Inspectorate under the license 2017-15-0201-01251.Tissue from human cerebral cortex was obtained at autopsy following Danish legislation concerning the use of human tissue for research.

\section{Consent to Participate Not applicable.}

Consent for Publication Not applicable.

Open Access This article is licensed under a Creative Commons Attribution 4.0 International License, which permits use, sharing, adaptation, distribution and reproduction in any medium or format, as long as you give appropriate credit to the original author(s) and the source, provide a link to the Creative Commons licence, and indicate if changes were made. The images or other third party material in this article are included in the article's Creative Commons licence, unless indicated otherwise in a credit line to the material. If material is not included in the article's Creative Commons licence and your intended use is not permitted by statutory regulation or exceeds the permitted use, you will need to obtain permission directly from the copyright holder. To view a copy of this licence, visit http://creativecommons.org/licenses/by/4.0/.

\section{References}

1. Masters CL, Simms G, Weinman NA, Multhaup G, McDonald BL, Beyreuther K (1985) Amyloid plaque core protein in Alzheimer disease and Down syndrome. Proc Natl Acad Sci U S A 82(12): 4245-4249. https://doi.org/10.1073/pnas.82.12.4245

2. Grundke-Iqbal I, Iqbal K, Tung Y-C, Quinlan M, Wisniewski H, Binder L (1987) Abnormal phosphorylation of the microtubuleassociated protein? (tau) in Alzheimer cytoskeletal pathology. Alzheimer Dis Assoc Disord 1(3):202. https://doi.org/10.1097/ 00002093-198701030-00020

3. Goedert M, Spillantini M, Cairns N, Crowther R (1992) Tau proteins of Alzheimer paired helical filaments: abnormal phosphorylation of all six brain isoforms. Neuron 8:159-168

4. Hardy J, Higgins G (1992) Alzheimer's disease: the amyloid cascade hypothesis. Science 256(5054):184-185

5. Hardy, J., \& Selkoe, D. J. (2002). The amyloid hypothesis of Alzheimer's disease: progress and problems on the road to therapeutics. science, 297(July).

6. Lambert J, Ibrahim-Verbaas C, Harold D, Naj A, Sims R, Bellenguez C et al (2013) Meta-analysis of 74,046 individuals identifies 11 new susceptibility loci for Alzheimer's disease. Nat Genet 45(12):1452-1458. https://doi.org/10.1038/ng.2802.Metaanalysis

7. Sims R, Hill M, Williams J (2020) The multiplex model of the genetics of Alzheimer's disease. Nat Neurosci 23(March):30-34. https://doi.org/10.1038/s41593-020-0599-5

8. Goate A, Chartier-Harlin MC, Mullan M, Brown J, Crawford F, Fidani L et al (1991) Segregation of a missense mutation in the amyloid precursor protein gene with familial Alzheimer's disease. Nature 349(6311):704-706. https://doi.org/10.1038/349704a0

9. Levy-lahad, E., Wasco, W., Poorkaj, P., Romano, D. M., Oshima, J., Pettingell, W. H., ... Tanzi, R. E. (1995). Candidate gene for the chromosome 1 familial Alzheimer's disease locus. Science, 269, 973-977.

10. Sherrington R, Rogaev EI, Liang Y, Rogaeva EA, Levesque G, Ikeda M, Chi H, Lin C et al (1995) Cloning of a gene bearing missense mutations in early-onset familial Alzheimer's disease. Nature. 375:754-760. https://doi.org/10.1038/375754a0

11. Sandbrink, R., Masters, C., \& Beyreuther, K. (1996). APP gene family. Alternative splicing generates functionally related isoforms. Annals of the New York Academy of Sciences, 777, 281-287. https://doi.org/10.1111/j.1749-6632.1996.tb34433.x

12. De Silva HAR, Jen A, Wickenden C, Jen LS, Wilkinson SL, Patel AJ (1997) Cell-specific expression of $\beta$-amyloid precursor protein isoform mRNAs and proteins in neurons and astrocytes. Mol Brain Res 47(1-2):147-156. https://doi.org/10.1016/S0169-328X(97) 00045-4

13. Mullan M, Crawford F, Axelman K, Houlden H, Lilius L, Winblad B, Lannfelt L (1992) A pathogenic mutation for probable Alzheimer's disease in the APP gene at the $\mathrm{N}$-terminus of $\beta$-amyloid. Nat Genet 1(5):345-347. https://doi.org/10.1038/ng0892-345

14. Murayama O, Tomita T, Nihonmatsu N, Murayama M, Sun X, Honda T, Iwatsubo T, Takashima A (1999) Enhancement of amyloid $\beta 42$ secretion by 28 different presenilin 1 mutations of familial Alzheimer's disease. Neurosci Lett 265(1):61-63. https://doi.org/ 10.1016/S0304-3940(99)00187-1

15. Goedert, M., Spillantini, M. C., Jakes, R., Rutherford, D., \& Crowther, R. A. (1989). Multiple 1soforms of human microtubule-associated protein tau: sequences and localization in 
neurofibrillah tangles of Alzheimer's disease. Neuron, 3(Table 1), 519-526.

16. Goedert M, Jakes R (1990) Expression of separate isoforms of human tau protein: correlation with the tau pattern in brain and effects on tubulin polymerization. EMBO J 9(13):4225-4230

17. Goedert M (2018) Tau filaments in neurodegenerative diseases. FEBS Lett 592:2383-2391. https://doi.org/10.1002/1873-3468. 13108

18. Tuerde D, Kimura T, Miyasaka T, Furusawa K, Shimozawa A, Hasegawa M, Ando K, Hisanaga S (2018) Isoform-independent and -dependent phosphorylation of microtubule-associated protein tau in mouse brain during postnatal development. J Biol Chem 293(26):1781-1793. https://doi.org/10.1074/jbc.M117.798918

19. Janke C, Beck M, Stahl T, Holzer M, Brauer K, Bigl V, Arendt T (1999) Phylogenetic diversity of the expression of the microtubuleassociated protein tau: implications for neurodegenerative disorders. Mol Brain Res 68(119-128):119-128

20. Dinkel PD, Siddiqua A, Huynh H, Shah M, Margittai M (2011) Variations in filament conformation dictate seeding barrier between three- and four-repeat tau. Biochemistry 50:4330-4336. https://doi. org/10.1021/bi2004685

21. Muratore CR, Rice HC, Srikanth P, Callahan DG, Shin T, Benjamin LNP et al (2014) The familial Alzheimer's disease APPV717I mutation alters APP processing and Tau expression in iPSC-derived neurons. Hum Mol Genet 23(13):3523-3536. https:// doi.org/10.1093/hmg/ddu064

22. Sposito T, Preza E, Mahoney CJ, Setó-salvia N, Ryan NS, Morris HR et al (2015) Developmental regulation of tau splicing is disrupted in stem cell-derived neurons from frontotemporal dementia patients with the $10+16$ splice-site mutation in MAPT. Hum Mol Genet 24(18):5260-5269. https://doi.org/10.1093/hmg/ ddv246

23. Correia AS, Rainone S, Potvin O, Kergoat M, Belleville S, Duchesne S (2019) Stem cell-derived neurons as cellular models of sporadic Alzheimer's disease. J Alzheimers Dis 67:893-910. https://doi.org/10.3233/JAD-180833

24. Verheyen A, Diels A, Reumers J, Van Hoorde K, Van Den Wyngaert I, Van Outryve C et al (2018) Genetically engineered iPSC-derived FTDP-17MAPT neurons display mutation-specific neurodegenerative and neurodevelopmental phenotypes. Stem Cell Reports 11(2):363-379. https://doi.org/10.1016/j.stemcr. 2018.06.022

25. Iovino M, Pfisterer U, Holton JL, Lashley T, Swingler RJ, Calo L et al (2014) The novel MAPT mutation K298E: mechanisms of mutant tau toxicity, brain pathology and tau expression in induced fibroblast-derived neurons. Acta Neuropathol 127:283-295. https:// doi.org/10.1007/s00401-013-1219-1

26. Espuny-camacho, I., Arranz, A. M., Fiers, M., Brion, J., Vanderhaeghen, P., Espuny-camacho, I., ... Radaelli, E. (2017). Hallmarks of Alzheimer's disease in stem-cell-derived human neurons transplanted into mouse brain. Neuron, 93(5), 1066-1081.e8. https://doi.org/10.1016/j.neuron.2017.02.001

27. Miguel L, Rovelet-lecrux A, Feyeux M, Frebourg T, Nassoy P, Campion D, Lecourtois M (2019) Detection of all adult Tau isoforms in a 3D culture model of iPSC-derived neurons. Stem Cell Res 40(January):101541. https://doi.org/10.1016/j.scr.2019. 101541

28. Habekost M, Jørgensen AL, Qvist P, Denham M (2020) MicroRNAs and Ascl1 facilitate direct conversion of porcine fibroblasts into induced neurons. Stem Cell Res 48:101984. https://doi. org/10.1016/j.scr.2020.101984

29. Mertens J, Paquola ACM, Ku M, Hatch E, Böhnke L, Ladjevardi S, McGrath S, Campbell B et al (2015) Directly reprogrammed human neurons retain aging-associated transcriptomic signatures and reveal age-related nucleocytoplasmic defects. Cell Stem Cell 17(6): 705-718. https://doi.org/10.1016/j.stem.2015.09.001
30. Birtele M, Sharma Y, Kidnapillai S, Lau S, Stoker TB, Barker A et al (2019) Dual modulation of neuron-specific microRNAs and the REST complex promotes functional maturation of human adult induced neurons. FEBS Lett 593:3370-3380. https://doi.org/10. 1002/1873-3468.13612

31. Jakobsen J, Johansen MG, Schmidt M, Dagnæs-Hansen F, Dam K, Gunnarsson A et al (2012) Generation of minipigs with targeted transgene insertion by recombinase-mediated cassette exchange (RMCE) and somatic cell nuclear transfer (SCNT). Transgenic Res 22(4):709-723. https://doi.org/10.1007/s11248-012-9671-6

32. Jakobsen JE, Johansen MG, Schmidt M, Liu Y, Li R, Callesen H, Melnikova M, Habekost M et al (2016) Expression of the Alzheimer's disease mutations A $\beta$ PP695sw and PSEN1M146I in double-transgenic göttingen minipigs. J Alzheimers Dis 53(4): 1617-1630. https://doi.org/10.3233/JAD-160408

33. Mandelkow E, Mandelkow E (2012) Biochemistry and cell biology of tau protein in neurofibrillary degeneration. Cold Spring Harbor perspectives in medicine: $1-25$

34. Patani R, Lewis P, Trabzuni D, Puddifoot C, Wyllie D, Walker R et al (2012) Investigating the utility of human embryonic stem cellderived neurons to model ageing and neurodegenerative disease using whole-genome gene expression and splicing analysis. J Neurochem 122(4):738-751. https://doi.org/10.1111/j.1471-4159. 2012.07825.x

35. Huh, C. J., Zhang, B., Victor, M. B., Dahiya, S., Batista, L. F. Z., Horvath, S., \& Yoo, A. S. (2016). Maintenance of age in human neurons generated by microRNA-based neuronal conversion of fibroblasts. eLife, 5(September2016), 1-14. https://doi.org/10.7554/ eLife.18648

36. Habekost M, Jørgensen AL, Qvist P, Denham M (2019) Transcriptomic profiling of porcine pluripotency identifies speciesspecific reprogramming requirements for culturing iPSCs. Stem Cell Res 41(February):101645. https://doi.org/10.1016/j.scr.2019.101645

37. Ezashi T, Yuan Y, Roberts RM (2016) Pluripotent stem cells from domesticated mammals. Annual Review of Animal Biosciences 4: 223-255. https://doi.org/10.1146/annurev-animal-021815-111202

38. Lacovich V, Espindola SL, Alloatti M, Devoto VP, Cromberg LE, Forte $\mathrm{G}$ et al (2017) Tau isoforms imbalance impairs the axonal transport of the amyloid precursor protein in human neurons. J Neurosci 37(1):58-69. https://doi.org/10.1523/JNEUROSCI.2305-16.2016

39. Heid CA, Stevens J, Livak KJ, Williams MP (1996) Real time quantitative PCR. Genome Res 6(10):986-994. https://doi.org/10. 1101/gr.6.10.986

40. Leinonen R, Sugawara H, Shumway M (2011) The sequence read archive. Nucleic Acids Res 39(SUPPL. 1):2010-2012. https://doi. org/10.1093/nar/gkq1019

41. Bolger AM, Lohse M, Usadel B (2014) Trimmomatic: a flexible trimmer for Illumina sequence data. Bioinformatics 30(15):2114 2120. https://doi.org/10.1093/bioinformatics/btu170

42. Kim D, Langmead B, Salzberg SL (2015) HISAT: a fast spliced aligner with low memory requirements. Nat Methods 12(4):357360. https://doi.org/10.1038/nmeth.3317

43. Liao Y, Smyth GK, Shi W (2014) FeatureCounts: an efficient general purpose program for assigning sequence reads to genomic features. Bioinformatics 30(7):923-930. https://doi.org/10.1093/ bioinformatics/btt656

44. Anders S, Reyes A, Huber W (2012) Detecting differential usage of exons from RNA-seq data. Genome Res 22:2008-2017. https://doi. org/10.1101/gr.133744.111.Freely

45. Love MI, Huber W, Anders S (2014) Moderated estimation of fold change and dispersion for RNA-seq data with DESeq2. Genome Biol 15(550):1-21. https://doi.org/10.1186/s13059-014-0550-8

Publisher's Note Springer Nature remains neutral with regard to jurisdictional claims in published maps and institutional affiliations. 